\title{
Superconducting quantum interference proximity transistor
}

\author{
Francesco Giazotto ${ }^{1 \star}$, Joonas T. Peltonen ${ }^{2}$, Matthias Meschke ${ }^{2}$ and Jukka P. Pekola ${ }^{2}$
}

\begin{abstract}
When a superconductor is placed close to a non-superconducting metal, it can induce superconducting correlations in the metal'10, known as the 'proximity effect'1". Such behaviour modifies the density of states (DOS) in the normal metal ${ }^{12-15}$ and opens a minigap $12,13,16$ with an amplitude that can be controlled by changing the phase of the superconducting order parameter ${ }^{12,15}$. Here, we exploit such behaviour to realize a new type of interferometer, the superconducting quantum interference proximity transistor (SQUIPT), for which the operation relies on the modulation with the magnetic field of the DOS of a proximized metal embedded in a superconducting loop. Even without optimizing its design, this device shows extremely low flux noise, down to $\sim 10^{-5} \Phi_{0} \mathrm{~Hz}^{-1 / 2}$ ( $\Phi_{0} \simeq 2 \times 10^{-15} \mathrm{~Wb}$ is the flux quantum) and dissipation several orders of magnitude smaller than in conventional superconducting interferometers ${ }^{17-19}$. With optimization, the SQUIPT could significantly increase the sensitivity with which small magnetic moments are detected.
\end{abstract}

One typical SQUIPT fabricated with electron-beam lithography is shown in Fig. 1a. It consists of an aluminium (Al) superconducting loop interrupted by a copper $(\mathrm{Cu})$ normal-metal wire in good electric contact with it. Furthermore, two $\mathrm{Al}$ electrodes are tunnel-coupled to the normal region to enable the device operation. A detailed view of the sample core (see Fig. $1 \mathrm{~b}$ ) shows the $\mathrm{Cu}$ region of length $L \simeq 1.5 \mu \mathrm{m}$ and width $\simeq 240 \mathrm{~nm}$ coupled to the tunnel probes and the superconducting loop. The SQUIPTs were implemented into two different designs (see Fig. 1c), namely, the A-type configuration, where the loop extends into an extra third lead, and the B-type configuration, which contains only two tunnel probes. The ring geometry enables us to change the phase difference across the normal-metal/superconductor boundaries through the application of an external magnetic field, which gives rise to a total flux $\Phi$ through the loop area. This modifies the DOS in the normal metal, and hence the transport through the tunnel junctions.

Insight into the interferometric nature of the SQUIPT can be gained by first analysing the theoretical prediction of its behaviour. Figure 2a shows the simplest implementation of the device in the A-type configuration, that is, that with just one junction tunnelcoupled to the normal metal. For simplicity, we suppose the tunnel probe (with resistance $R_{\mathrm{T}}$ ) to be placed in the middle of the wire, and to feed a constant electric current $I$ through the circuit while the voltage drop $V$ is recorded as a function of $\Phi$. In the limit that the kinetic inductance of the superconducting loop is negligible, the magnetic flux fixes a phase difference $\phi=2 \pi \Phi / \Phi_{0}$ across the normal metal, where $\Phi_{0}=\pi \hbar / e$ is the flux quantum, $\hbar$ is the reduced Planck's constant and $e$ is the electron charge. Figure $2 \mathrm{~b}$ shows the low-temperature quasiparticle current-voltage $(I-V)$ characteristic of the SQUIPT calculated at a few selected values of $\Phi$. The calculations were carried out for parameters similar to those

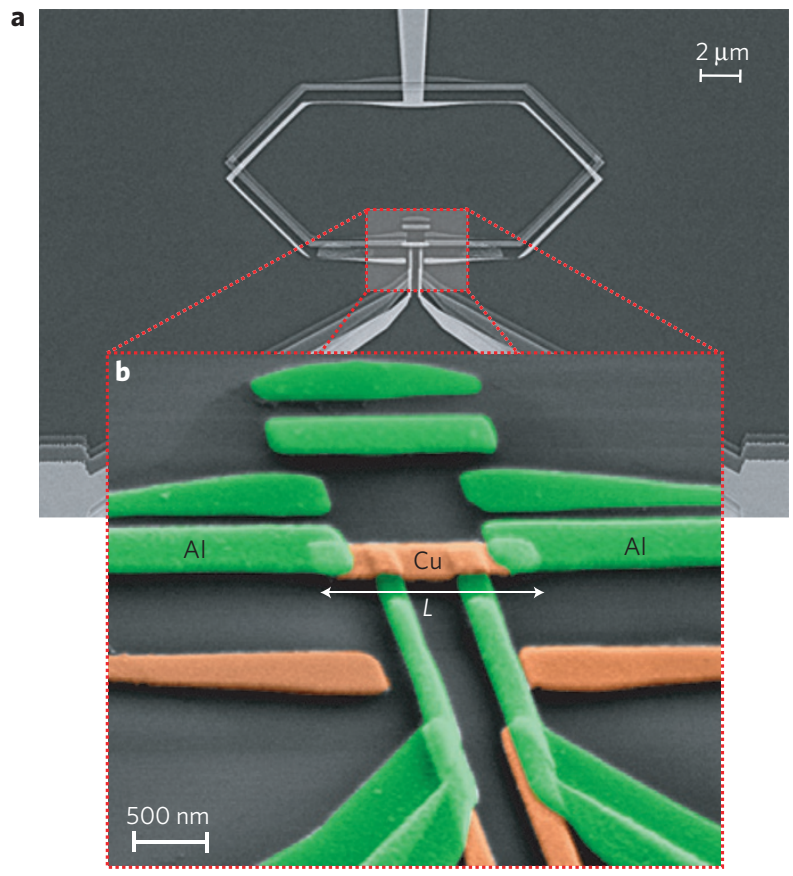

C

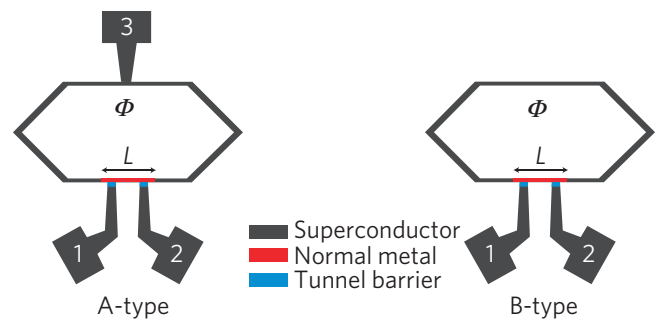

Figure 1 | The SQUIPT. a, Scanning electron micrograph of a typical SQUIPT. An Al superconducting ring is interrupted by a normal-metal $\mathrm{Cu}$ wire in good electric contact with it. Two Al tunnel junctions (bottom of the image) are connected to the normal metal to enable the device operation. b, A pseudo-colour detailed view of the junction region showing the $\mathrm{Al} / \mathrm{Cu} / \mathrm{Al}$ superconductor/normal-metal/superconductor proximity junction, as well as the two Al electrodes (of width $\sim 200 \mathrm{~nm}$ ) connected by means of an $\mathrm{AlO}_{x}$ tunnel barrier to the $\mathrm{Cu}$ wire. The $\mathrm{Cu}$ wire is overlapped laterally by the Al leads for about $250 \mathrm{~nm}$. The structure replicas resulting from the shadow-mask fabrication procedure are visible. c, Schematic of the two device geometries investigated. The superconducting ring extends into an extra third lead in the A-type configuration. The electrodes labelled 1, 2 and 3 are used to operate the SQUIPT. $L \simeq 1.5 \mu \mathrm{m}$ denotes the total length of the normal-metal region and $\Phi$ is the applied magnetic flux threading the loop.

${ }^{1}$ NEST Istituto Nanoscienze-CNR and Scuola Normale Superiore, Piazza S. Silvestro 12, I-56127 Pisa, Italy, ${ }^{2}$ Low Temperature Laboratory, Aalto University School of Science and Technology, PO Box 13500, Fl-00076 Aalto, Finland. *e-mail: giazotto@sns.it. 


\section{a}
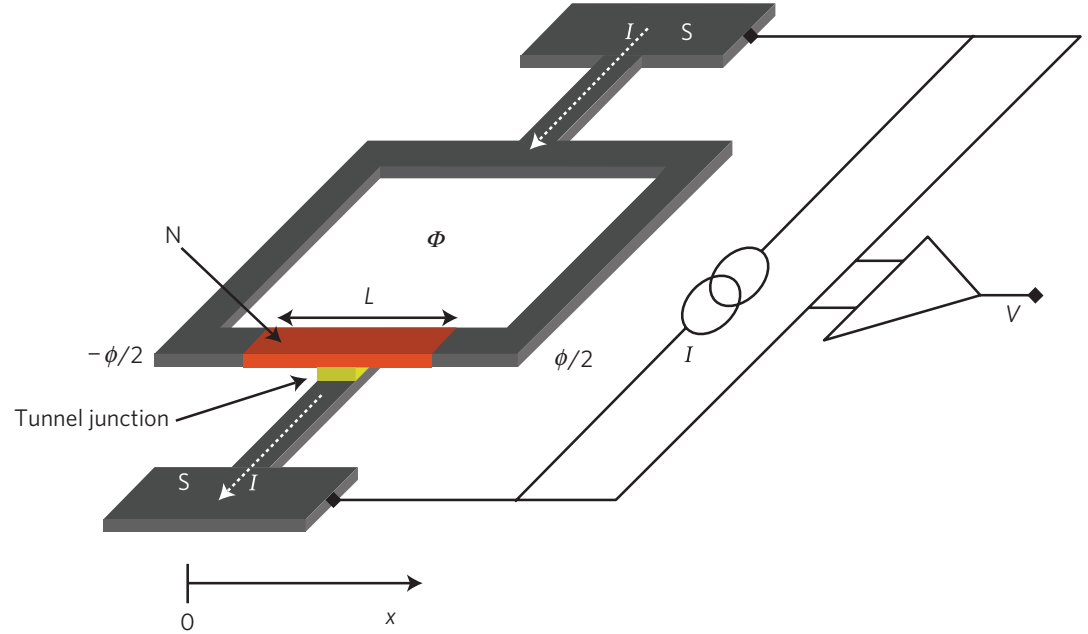

b

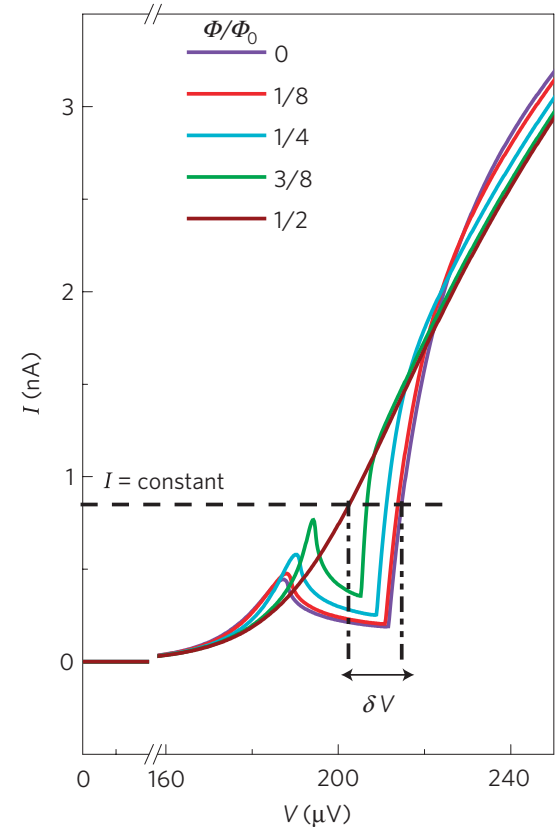

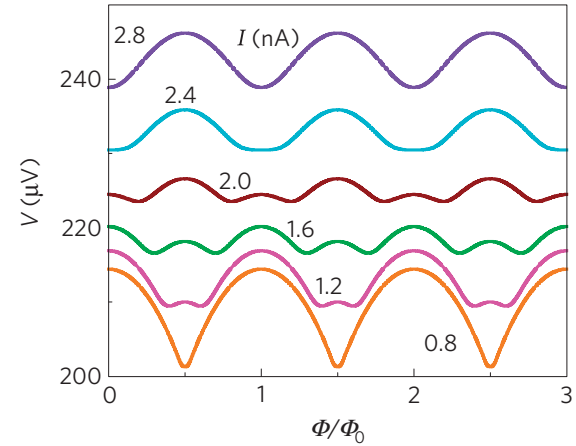

d

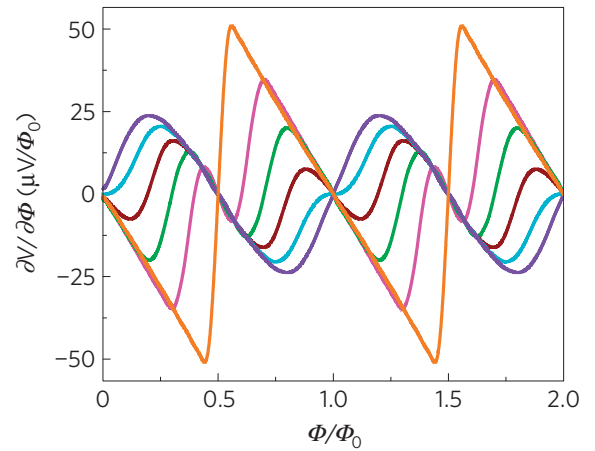

Figure 2 | SQUIPT configuration and its predicted behaviour. a, Schematic of the SQUIPT implemented in the A-type configuration. The superconducting (S) tunnel probe with normal-state resistance $R_{\mathrm{T}}$ is placed in the middle of the normal-metal $(\mathrm{N})$ wire, that is, at $x=0$. An electric current $I$ is fed into the circuit, and the voltage drop $V$ is recorded as a function of the applied magnetic flux $\Phi$. $\phi$ is the macroscopic quantum phase of the superconducting order parameter. b, SQUIPT quasiparticle current-voltage characteristics calculated for some values of the applied flux $\Phi$. When biased with a constant current $I$, a voltage modulation $V(\Phi)$ with amplitude $\delta V$ appears across the device as a function of the applied magnetic flux. c, Voltage modulation $V(\Phi)$ for some values of the bias current. d, Flux-to-voltage transfer function $\partial V / \partial \Phi$ calculated for the same current values as in $\mathbf{c}$. All calculations were carried out at temperature $T=0.1 T_{\mathrm{c}}$ by setting $E_{\mathrm{Th}}=4 \mu \mathrm{eV}$, which is similar to that in our devices. Here, $T_{\mathrm{c}} \simeq 1.3 \mathrm{~K}$ is the superconducting critical temperature, $E_{\mathrm{Th}}=\hbar D / L^{2}$ is the Thouless energy, that is, the characteristic energy scale of the normal-metal region and $D=110 \mathrm{~cm}^{2} \mathrm{~s}^{-1}$ is the electron diffusion constant in $\mathrm{Cu}$. Furthermore, we set $\Delta_{0}=200 \mu \mathrm{eV}$ as the zero-temperature Al superconducting gap and $R_{\mathrm{T}}=50 \mathrm{k} \Omega$.

of our structures ${ }^{20}$. Whereas for $\Phi=0$, that is, when the minigap in the normal metal is maximized ${ }^{15,16}$, the characteristic resembles that of a superconductor-insulator-superconductor junction $^{18}$, for $\Phi=\Phi_{0} / 2$ the characteristic corresponds to that of a normal-metal/insulator/superconductor contact, with the minigap suppressed $^{15,16}$. The SQUIPT thus behaves as a flux-to-voltage transformer for which the response $V(\Phi)$ (and amplitude $\delta V$ ) depends on the bias current $I$ through the tunnel junction. The interferometer voltage modulation $V(\Phi)$ is shown in Fig. 2c for different values of $I$. In particular, $V(\Phi)$ is strongly dependent on the bias current, the latter determining the exact shape of the device response. Note the change of concavity of $V(\Phi)$, which occurs as the bias current exceeds the point where the $I-V$ characteristics cross. One relevant figure of merit of the SQUIPT is represented by the flux-to-voltage transfer function, $\partial V / \partial \Phi$, which is shown in Fig. $2 \mathrm{~d}$. It turns out that $\partial V / \partial \Phi$ is a non-monotonic function of the bias current, as well as its sign depending on the specific value of $I$.

Figure 3a shows the experimental low-temperature $I-V$ characteristic of a device implemented in the A-type configuration. The curve resembles that of a typical superconducting tunnel junction, where the onset of large quasiparticle current is set by the energy gap $\left(\Delta_{0} \simeq 200 \mu \mathrm{eV}\right.$ in our samples). The absence of the peak in the experimental curves, as compared with the theoretical ones in Fig. 2b, could originate from broadening resulting from inelastic 


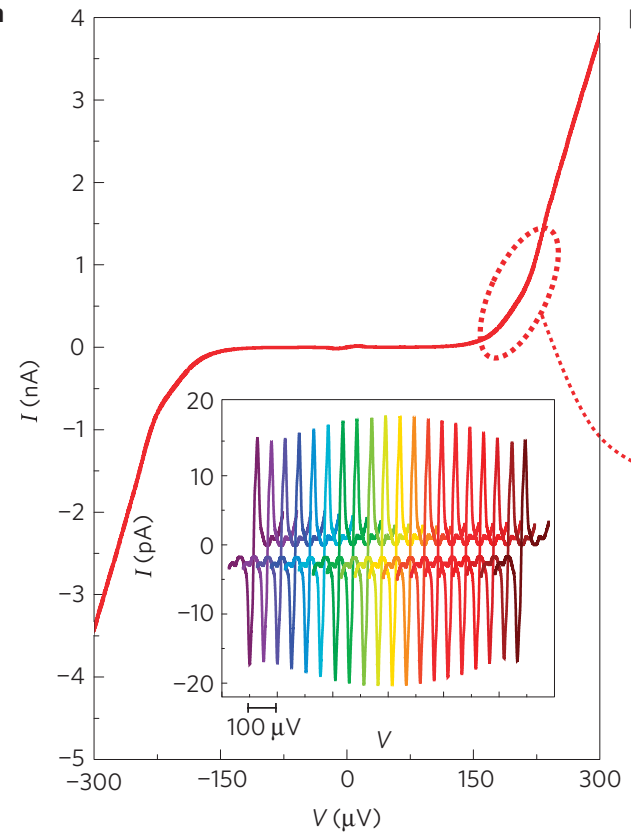

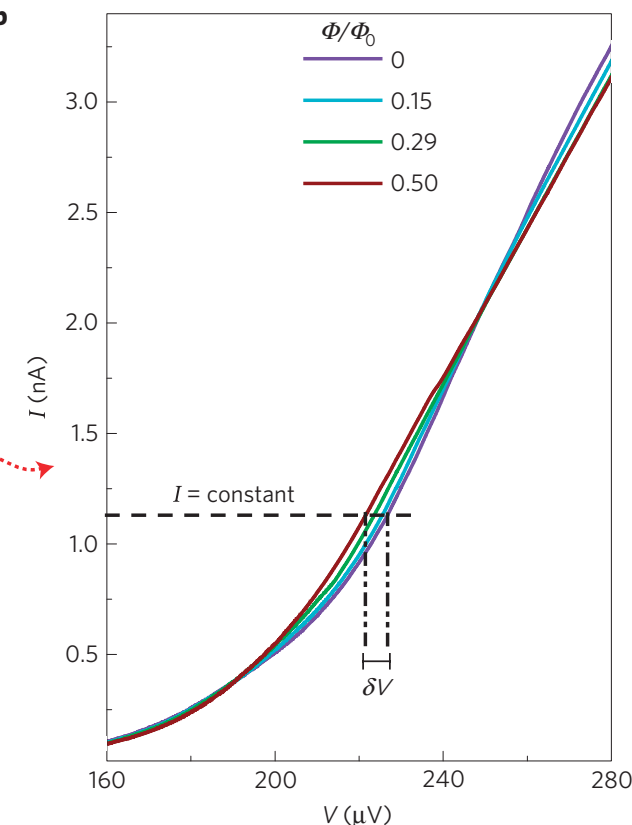

Figure 3 | Magnetic-field dependence of the current-voltage characteristic. a, Current-voltage characteristic of an A-type SQUIPT with normal-state resistance $R_{\mathrm{T}} \simeq 50 \mathrm{k} \Omega$ measured at $68 \mathrm{mK}$. The inset shows the enlargement around zero bias of the characteristic of the same device measured at $53 \mathrm{mK}$ for several applied magnetic-field values. The peak appearing around zero bias, and with magnitude $I \simeq 17 \mathrm{pA}$, is the Josephson current flowing through the structure, and its magnitude shows a periodic modulation over a magnetic flux quantum $\Phi_{0}$. The curves are horizontally offset for clarity, and correspond to a magnetic-field intensity increasing with a step of $\sim 10^{-2}$ Oe. For the present structure, $\Phi_{0}$ corresponds to an applied field $B=\Phi_{0} / A \simeq 0.17$ Oe, where $A \simeq 120 \mu \mathrm{m}^{2}$ is the loop area. $\mathbf{b}$, Detailed view of the current-voltage characteristic of the same device for larger bias voltages measured at $53 \mathrm{mK}$ for some values of the applied magnetic flux $\Phi$ up to $\Phi_{0} / 2$. Also shown is the voltage modulation amplitude $\delta V$ occurring when biasing the SQUIPT with a constant current $I$. All measurements on the A-type structure were carried out through electrodes 1 and 3 (see Fig. 1c)

scattering or finite quasiparticle lifetime in the superconductor ${ }^{21}$. A deeper inspection reveals, however, that the characteristic is modulated by the presence of an applied magnetic field. The effect is clearly visible in Fig. 3b, which shows a detailed view of the curve at large bias voltage for some values of the applied flux up to $\Phi_{0} / 2$. Such a modulation is of coherent nature, and stems from magnetic-field-induced control of the DOS in the normal metal. In addition to the quasiparticle current, a Josephson coupling is observed at the lowest temperatures, and manifests itself as a peak around zero bias in the $I-V$ characteristic (see the inset of Fig. 3a). The supercurrent, which is expected to exist in proximized structures such as the present one $e^{12}$, obtains values as high as $\simeq 17 \mathrm{pA}$ at $53 \mathrm{mK}$. It is modulated by the applied flux with the same periodicity as for the quasiparticle current.

The full $V(\Phi)$ dependence for the same A-type sample at several values of the bias current is shown in Fig. 4a. As expected (see Fig. 2c), the modulation amplitude $\delta V$ is a non-monotonic function of $I$, and $V(\Phi)$ shows a change of concavity whenever the bias current exceeds the crossing points of the current-voltage characteristic (see Fig. 3b). In this sample, $\delta V$ obtains values as large as $\sim 7 \mu \mathrm{V}$ at $1 \mathrm{nA}$. The corresponding transfer function is shown in Fig. $4 \mathrm{~b}$ for a few bias currents. A resemblance to the theoretical prediction of Fig. $2 c, d$ is obvious. In such a case, $|\partial V / \partial \Phi|$ as large as $\simeq 30 \mu \mathrm{V} / \Phi_{0}$ is obtained at $1 \mathrm{nA}$. The maximum of $|\partial V / \partial \Phi|$ for the same SQUIPT is shown in Fig. 4c, and highlights the expected non-monotonic dependence on $I$.

Figure $4 \mathrm{~d}$ and e show $V(\Phi)$ and the maximum of $|\partial V / \partial \Phi|$, respectively, for a B-type SQUIPT. $\delta V$ obtains in this case values as high as $\sim 12 \mu \mathrm{V}$ at $1 \mathrm{nA}$, and $|\partial V / \partial \Phi|$ is maximized at $0.6 \mathrm{nA}$ where it reaches $\simeq 60 \mu \mathrm{V} / \Phi_{0}$. We emphasize that these values are larger by almost a factor of two than those obtained in the A-type device. This is to be expected because in a B-type sample, $V(\Phi)$ is probed across two tunnel junctions in series. This doubles the
SQUIPT response. The above results are roughly $50-60 \%$ of those predicted by our calculations (see Fig. 2), which can be ascribed either to the uncertainty in the precise determination of the device parameters ${ }^{20}$ or to non-ideal phase biasing of the interferometers ${ }^{15}$. In the limit of negligible geometric inductance of the loop $(\sim 40 \mathrm{pH}$ for our rings), a phase difference $\phi=2 \pi \Phi / \Phi_{0}$ can be induced across the normal metal if the phase accumulated in the superconductor is much smaller than that accumulated in the wire, that is, if the ratio between their respective kinetic inductances is much smaller than unity. We estimate the kinetic inductance of the superconducting loop to be of the order of $\sim 150 \mathrm{pH}$, whereas that of the wire to be around $\sim 300-500 \mathrm{pH}$, so that the correction factor to the actual phase bias determined by such a ratio $(\sim 0.3-0.5$ for our devices) may prevent the full closing of the minigap, thus weakening the SQUIPT response.

The role of temperature $(T)$ is shown in Fig. 5a, which shows $V(\Phi)$ at $1 \mathrm{nA}$ for several increasing temperatures for a B-type sample. $\delta V$ initially monotonically decreases with increasing $T$ up to $\sim 400 \mathrm{mK}$, then it starts to increase again and it is almost suppressed at $730 \mathrm{mK}$. The full temperature dependence of the maximum of $|\partial V / \partial \Phi|$ at $1 \mathrm{nA}$ is shown in Fig. $5 \mathrm{~b}$ for the same device, and reflects the above non-monotonic behaviour similarly to that observed for different bias currents (see Fig. 4c).

Compared with conventional d.c. superconducting quantum interference devices ${ }^{17-19}$ (SQUIDs), power dissipation $(P)$ is markedly suppressed in the SQUIPT. In our devices we have $P \sim 10^{2} \mathrm{fW}$, which can be further reduced by simply increasing the resistance of the probing junctions. This power is four-five orders of magnitude smaller than that in conventional d.c. SQUIDs, which makes the SQUIPT ideal for applications where very low dissipation is required.

We shall finally comment on another figure of merit of the SQUIPT, namely, its noise-equivalent flux (NEF) or 'flux 


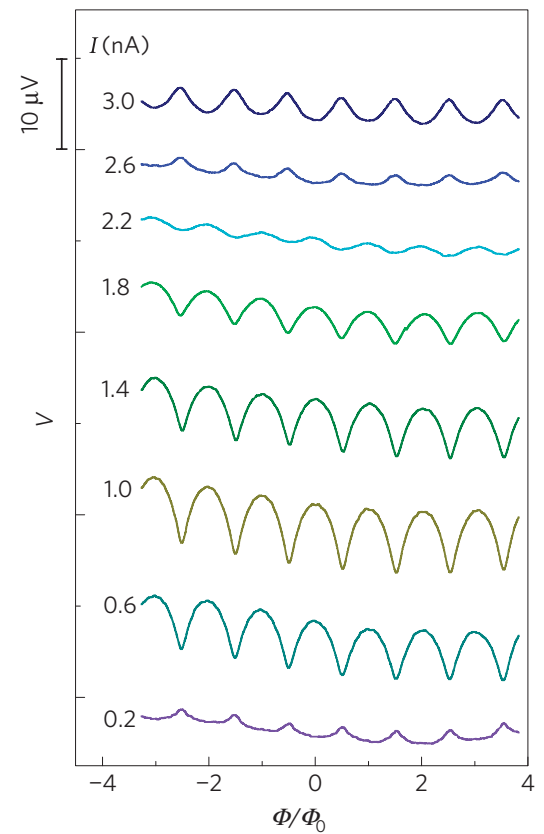

d

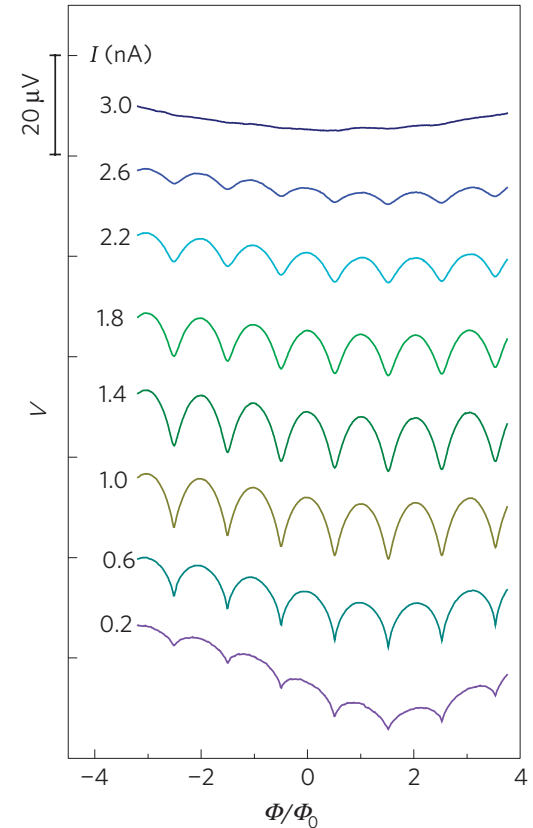

b

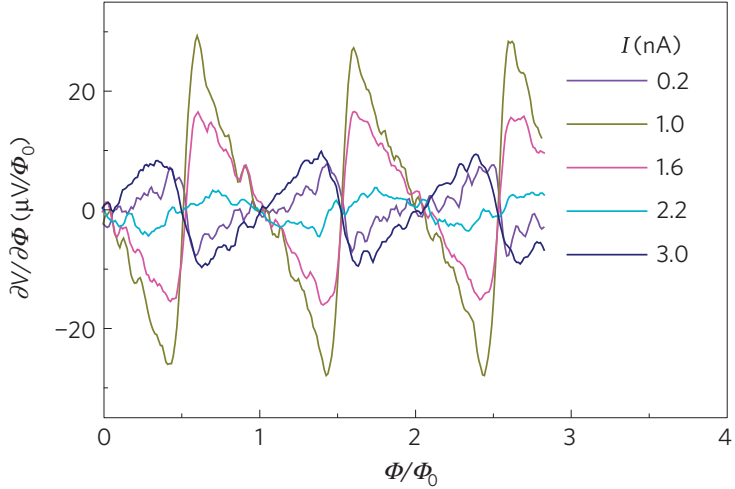

C

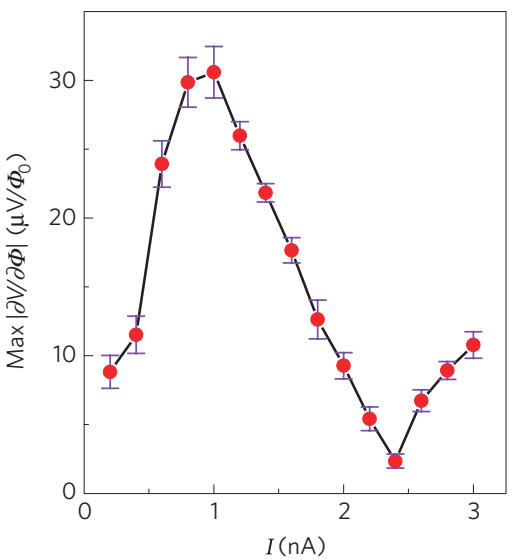

e

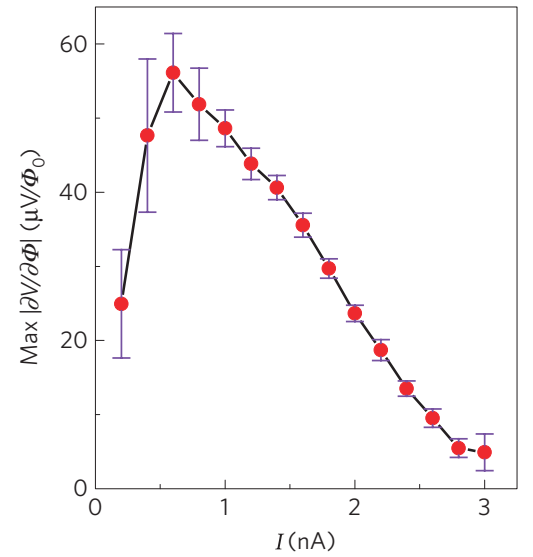

Figure 4 | Magnetic-field dependence of the voltage modulation and flux-to-voltage transfer function. a, Voltage modulation $V(\Phi)$ of an A-type structure measured at $54 \mathrm{mK}$ for several values of the bias current $I$. For the present device, $\delta V$ obtains values as large as $\sim 7 \mu \mathrm{V}$ at $1 \mathrm{nA}$. Note the change of concavity of $V(\Phi)$ for different values of $I$. The curves are vertically offset for clarity. b, Flux-to-voltage transfer function $\partial V / \partial \Phi$ of the same A-type structure at $54 \mathrm{mK}$ at selected bias currents. The transfer functions were obtained by numerically differentiating the $V(\Phi)$ curves. c, Maximum value of $|\partial V / \partial \Phi|$ versus injection current for an A-type structure at $54 \mathrm{mK}$. d, Voltage modulation $V(\Phi)$ of a B-type structure measured at $53 \mathrm{mK}$ for several values of the bias current $I$. For this device, the maximum amplitude of the voltage modulation is $\delta V \sim 12 \mu \mathrm{V}$ at $1 \mathrm{nA}$. The curves are vertically offset for clarity. e, Maximum value of $|\partial V / \partial \Phi|$ versus injection current for the same B-type structure at $53 \mathrm{mK}$. Measurements on the B-type structure were carried out through electrodes 1 and 2 (see Fig. 1c). The error bars shown in $\mathbf{c}$ and $\mathbf{e}$ represent the standard deviation of the maximum values numerically calculated over several flux periods.

sensitivity', defined as $\mathrm{NEF}=\left\langle V_{\mathrm{N}}^{2}\right\rangle^{1 / 2} /|\partial V / \partial \Phi| \delta \nu^{1 / 2}$ (ref. 19), where $V_{\mathrm{N}}$ is the voltage noise of the interferometer within the frequency band $\delta \nu$. In our experiment, we can provide an upper estimate for NEF, because it is believed to be limited mainly by the preamplifier noise. With a typical r.m.s. noise of $\sim 1.2 \mathrm{nV} \mathrm{Hz}^{-1 / 2}$ in our set-up, we estimate $\mathrm{NEF} \simeq 2 \times 10^{-5} \Phi_{0} \mathrm{~Hz}^{-1 / 2}$ at best (with the onset of $1 / f$ noise roughly below $\sim 1 \mathrm{~Hz}$ ), which should be substantially higher than the SQUIPT intrinsic NEF. Assuming that the noise of the interferometer is essentially of Johnson type, the intrinsic $V_{\mathrm{N}}$ can be estimated by noting that in the range of experimental bias currents the device dynamic resistance is typically within the range $\sim 25-70 \mathrm{k} \Omega$, leading to $V_{\mathrm{N}} \sim 0.3-0.5 \mathrm{nV} \mathrm{Hz}^{-1 / 2}$ and $\mathrm{NEF} \simeq 5-8 \mu \Phi_{0} \mathrm{~Hz}^{-1 / 2}$ at $54 \mathrm{mK}$. We note that the preamplifier contribution to the noise can be made negligible by increasing $|\partial V / \partial \Phi|$, that is, by optimizing the SQUIPT parameters (for instance, by choosing a superconducting material with a larger gap 


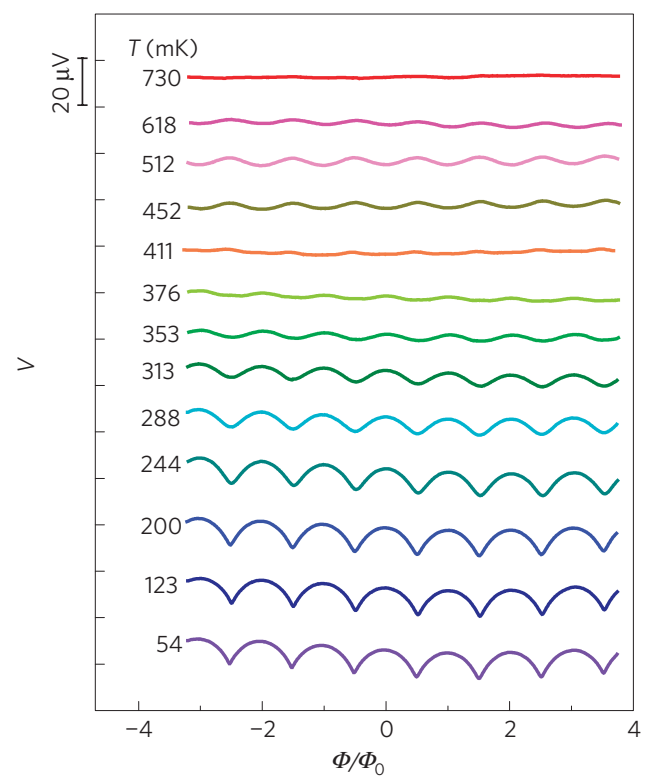

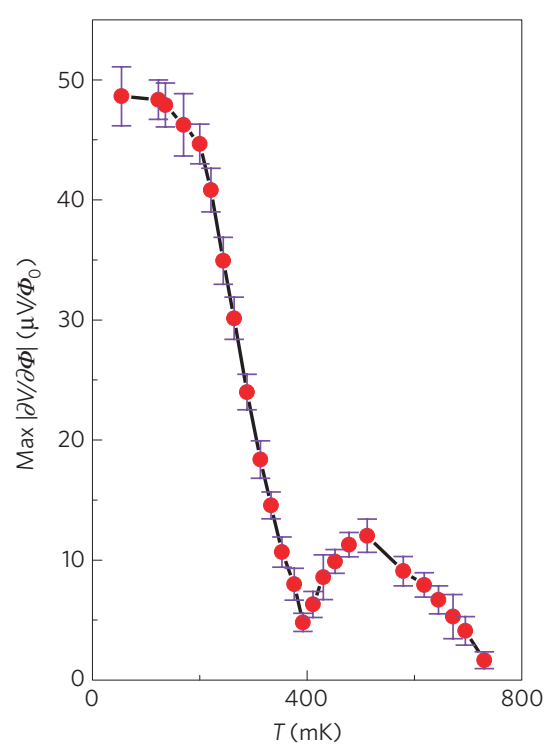

Figure 5 | Temperature dependence of the voltage modulation and flux-to-voltage transfer function. a, Voltage modulation $V(\Phi)$ of the $B$-type structure measured at several bath temperatures for $I=1 \mathrm{nA} . V(\Phi)$ changes concavity by increasing the temperature between 376 and $411 \mathrm{mK}$. The curves are vertically offset for clarity. $\mathbf{b}$, Maximum value of $|\partial \mathrm{V} / \partial \Phi|$ versus temperature for the same device at $I=1 \mathrm{nA}$. This behaviour resembles in part that of Fig. 4c observed for different bias current values. The error bars represent the standard deviation of the maximum values numerically calculated over several flux periods.

and by making a shorter normal-metal wire) and the phase bias as well. Our calculations show that by replacing niobium $(\mathrm{Nb})$ as the superconductor (with $\Delta_{0} \simeq 1.5 \mathrm{meV}$ ), and by shortening $L$ down to $150 \mathrm{~nm},|\partial V / \partial \Phi|$ as large as $\sim 2.5 \mathrm{mV} / \Phi_{0}$ could be achieved, yielding a preamplifier $\mathrm{NEF} \simeq 4 \times 10^{-7} \Phi_{0} \mathrm{~Hz}^{-1 / 2}$. The device intrinsic noise deserves however further investigation.

The SQUIPT has a number of features that make it attractive for a variety of applications: (1) only a simple d.c. read-out scheme is required, similarly to d.c. SQUIDs; (2) either currentor voltage-biased measurement can be conceived depending on the set-up requirements; (3) a large flexibility in the fabrication parameters and materials, such as semiconductors ${ }^{6,9}$, carbon nanotubes ${ }^{2,3,5}$ or graphene $e^{1}$ instead of normal metals, is allowed to optimize the response and the operating temperature (to this end superconducting $V$ (ref. 22) or $\mathrm{Nb}$ are suitable candidates); (4) ultralow dissipation $(\sim 1 \ldots 100 \mathrm{fW})$, which makes it ideal for nanoscale applications; (5) ease of implementation in a series or parallel array (depending on the biasing mode) for enhanced output; and (6) ease of integration with superconducting refrigerators ${ }^{23}$ to actively tune the device working temperature. Finally, as far as specific applications ${ }^{24}$ are concerned, measurements of small magnetic systems ${ }^{25}$ (for instance, the magnetic flux induced by atomic spins as well as singlemolecule nanomagnets), single-photon detection ${ }^{26}$, scanning microscopy as well as quantum metrology ${ }^{27}$ and nanoelectromechanical measurements ${ }^{28}$ with SQUIPT devices could be predicted. Our approach opens the way to magnetic-field detection based on 'hybrid' interferometers that take advantage of the flexibility intrinsic to proximity metals.

\section{Methods}

Fabrication details and experimental set-up. The samples were fabricated at the Low Temperature Laboratory at Aalto University School of Science and Technology by standard three-angle shadow-mask evaporation of the metals through a conventional suspended resist mask in a single vacuum cycle. Initially, a bilayer poly(methyl methacrylate)/copolymer resist was spun on an oxidized Si wafer, onto which the structures were patterned using electron-beam lithography. In the electron-gun evaporator, the chip was first tilted to an angle of $25^{\circ}$ with respect to the source, and approximately $27 \mathrm{~nm}$ of $\mathrm{Al}$ was evaporated to create the superconducting electrodes of the probe tunnel junctions. To form the tunnel barriers, the sample was exposed to 4.4 mbar of oxygen for five minutes, and consequently tilted to $-25^{\circ}$ for the deposition of $27 \mathrm{~nm}$ of $\mathrm{Cu}$ forming the normal-metal island. Immediately after this, the chip was tilted to the final angle of $9^{\circ}$, and $60 \mathrm{~nm}$ of $\mathrm{Al}$ was deposited to form the superconducting loop and the transparent normal-metal/superconductor contacts. The magneto-electric characterization was carried out at NEST Istituto Nanoscienze-CNR by cooling the devices with a filtered ${ }^{3} \mathrm{He} /{ }^{4} \mathrm{He}$ dilution refrigerator down to $\simeq 50 \mathrm{mK}$. Current and voltage were measured with room-temperature preamplifiers.

Theoretical model. The considered system consists of a one-dimensional diffusive normal-metal wire of length $L$ in good electric contact with two superconducting leads, which define a ring (see Fig. 2a). The contact with the superconductors enables superconducting correlations to be induced into the normal-metal region through the proximity effect ${ }^{11}$, which is responsible for the modification of the DOS in the wire ${ }^{13}$, as well as for the Josephson current to flow through the superconductor/normal-metal/superconductor structure ${ }^{12}$. The proximity effect in the normal-metal region of the SQUIPT can be described with the Usadel equations ${ }^{29}$, which can be written as ${ }^{12,29}$

$$
\begin{aligned}
\hbar D \partial_{x}^{2} \theta= & -2 i E \sinh (\theta)+\frac{\hbar D}{2}\left(\partial_{x} \chi\right)^{2} \sinh (2 \theta) \\
& \sinh (2 \theta) \partial_{x} \theta \partial_{x} \chi+\sinh ^{2}(\theta) \partial_{x}^{2} \chi=0
\end{aligned}
$$

where $D$ is the diffusion constant and $E$ is the energy relative to the chemical potential in the superconductors. $\theta$ and $\chi$ are, in general, complex scalar functions of position $x$ and energy. For perfectly transmitting interfaces, the boundary conditions at the normal-metal/superconductor contacts (that is, $x= \pm L / 2$, see Fig. 2a) reduce to $\theta( \pm L / 2)=\operatorname{arctanh}(\Delta / E)$ and $\chi( \pm L / 2)= \pm \phi / 2$, where $\phi$ is the phase difference across the normal-metal/superconductor boundaries and $\Delta$ is the superconducting order parameter. For simplicity, we chose a step-function form for the order parameter, that is, constant in the superconductor and zero in the normal-metal region, although $\Delta$ is in principle position dependent and can be determined self-consistently ${ }^{12}$. The DOS in the normal-metal region normalized to the DOS at the Fermi level in the absence of the proximity effect is given by $N_{\mathrm{N}}(x, E, \phi)=\operatorname{Re}\{\cosh [\theta(x, E, \phi)]\}$. From the numerical solution of equations (1), we get the DOS as a function of position and energy for any given $\phi$. In particular, the DOS is an even function of energy, and has a minigap $\left(E_{\mathrm{g}}\right)$ for $|E| \leq E_{\mathrm{g}}$ (ref. 13) with a magnitude that depends in general on the Thouless energy $E_{\mathrm{Th}}=\hbar D / L^{2}$, the characteristic energy scale for the normal region, and on $\phi$. The minigap $E_{\mathrm{g}}$ is maximum for $\phi=0$ and decreases with increasing $\phi$, vanishing at $\phi=\pi$ (ref. 16; the behaviour is $2 \pi$-periodic in $\phi$ ). 
The quasiparticle current $\left(I_{\mathrm{qp}}\right)$ through the superconducting tunnel junction biased at voltage $V$ (see Fig. 2a) can be calculated from ${ }^{30}$

$$
I_{\mathrm{qp}}(x, \phi, T)=\frac{1}{e R_{\mathrm{T}}} \int_{-\infty}^{\infty} \mathrm{d} E N_{\mathrm{S}}(E-e V) N_{\mathrm{N}}(x, E, \phi)\left[f_{0}(E-e V)-f_{0}(E)\right]
$$

where $e$ is the electron charge, $R_{\mathrm{T}}$ is the tunnel junction resistance, $N_{\mathrm{S}}(E)=|E| / \sqrt{E^{2}-\Delta^{2}} \Theta\left(E^{2}-\Delta^{2}\right)$ is the Bardeen-Cooper-Schrieffer-normalized DOS in the superconductor, $\Theta(z)$ is the Heaviside step function, $f_{0}(E)=\left(1+\exp \left[E /\left(k_{\mathrm{B}} T\right)\right]\right)^{-1}$ is the Fermi-Dirac distribution function at temperature $T$ and $k_{\mathrm{B}}$ is the Boltzmann constant. For any bias current $\left(I_{\text {bias }}\right)$ imposed across the SQUIPT, the voltage response $V(\phi)$ is determined from the solution of the equation $I_{\text {bias }}-I_{\mathrm{qp}}(x, \phi, T)=0$. From this, we calculate the flux-to-voltage transfer function $\partial V / \partial \phi$.

\section{Received 1 December 2009; accepted 27 January 2010;} published online 28 February 2010; corrected online 10 June 2010

\section{References}

1. Heersche, H. B., Jarillo-Herrero, P., Oostinga, J. B., Vandersypen, L. M. K. \& Morpurgo, A. F. Bipolar supercurrent in graphene. Nature 446, 56-59 (2007).

2. Jarillo-Herrero, P., van Dam, J. A. \& Kouwenhoven, L. P. Quantum supercurrent transistors in carbon nanotubes. Nature 439, 953-956 (2006).

3. Morpurgo, A. F., Kong, J., Marcus, C. M. \& Dai, H. Gate-controlled superconducting proximity effect in carbon nanotubes. Science 286, 263-265 (1999).

4. Kasumov, A. Yu. et al. Proximity-induced superconductivity in DNA. Science 291, 280-282 (2001).

5. Cleuziou, J.-P., Wernsdorfer, W., Bouchiat, V., Ondarcuhu, T. \& Monthioux, $\mathrm{M}$. Carbon nanotube superconducting quantum interference device. Nature Nanotech. 1, 53-59 (2006).

6. Doh, Y.-J. et al. Tunable supercurrent through semiconductor nanowires. Science 309, 272-275 (2005).

7. Pothier, H., Guéron, S., Esteve, D. \& Devoret, M. H. Flux-modulated Andreev current caused by electronic interference. Phys. Rev. Lett. 73, 2488-2491 (1994).

8. Courtois, H., Gandit, Ph. \& Pannetier, B. Proximity-induced superconductivity in a narrow metallic wire. Phys. Rev. B 52, 1162-1166 (1995).

9. Giazotto, F. et al. Resonant transport in Nb/GaAs/AlGaAs heterostructures: Realization of the de Gennes-Saint-James model. Phys. Rev. Lett. 87, 216808 (2001).

10. Baselmans, J. J. A., Morpurgo, A. F., van Wees, B. J. \& Klapwijk, T. M. Reversing the direction of the supercurrent in a controllable Josephson junction. Nature 397, 43-45 (1999).

11. de Gennes, P. G. Superconductivity of Metals and Alloys (W. A. Benjamin, 1966).

12. Belzig, W., Wilhelm, F. K., Bruder, C., Schön, G. \& Zaikin, A. D. Quasiclassical Greens function approach to mesoscopic superconductivity. Superlattices Microstruct. 25, 1251-1288 (1999).

13. Belzig, W., Bruder, C. \& Schön, G. Local density of states in a dirty normal metal connected to a superconductor. Phys. Rev. B 54, 9443-9448 (1996).

14. Guéron, S., Pothier, H., Birge, N. O., Esteve, D. \& Devoret, M. H. Superconducting proximity effect probed on a mesoscopic length scale. Phys. Rev. Lett. 77, 3025-3028 (1996).
15. le Sueur, H., Joyez, P., Pothier, H., Urbina, C. \& Esteve, D. Phase controlled superconducting proximity effect probed by tunneling spectroscopy. Phys. Rev. Lett. 100, 197002 (2008).

16. Zhou, F., Charlat, P., Spivak, B. \& Pannetier, B. Density of states in superconductor-normal metal-superconductor junctions. J. Low Temp. Phys. 110, 841-850 (1998).

17. Clarke, J. \& Braginski, A. I. (eds) The SQUID Handbook (Wiley-VCH, 2004).

18. Tinkham, M. Introduction to Superconductivity 2nd edn (McGraw-Hill, 1996).

19. Likharev, K. K. Dynamics of Josephson Junctions and Circuits (Gordon and Breach, 1986).

20. Courtois, H., Meschke, M., Peltonen, J. T. \& Pekola, J. P. Origin of hysteresis in a proximity Josephson junction. Phys. Rev. Lett. 101, 067002 (2008).

21. Timofeev, A. V. et al. Recombination-limited energy relaxation in a Bardeen-Cooper-Schrieffer superconductor. Phys. Rev. Lett. 102, 017003 (2009).

22. Pascual García, C. \& Giazotto, F. Josephson current in nanofabricated V/Cu/V mesoscopic junctions. Appl. Phys. Lett. 94, 132508 (2009).

23. Giazotto, F., Heikkilá, T. T., Luukanen, A., Savin, A. M. \& Pekola, J. P. Opportunities for mesoscopics in thermometry and refrigeration: Physics and applications. Rev. Mod. Phys. 78, 217-274 (2006).

24. Foley, C. P. \& Hilgenkamp, H. Why nanoSQUIDs are important: An introduction to the focus issue. Supercond. Sci. Technol. 22, 1-5 (2009).

25. Raufast, C. et al. Microwave-assisted magnetization reversal in individual isolated clusters of cobalt. IEEE Trans. Magn. 44, 2812-2815 (2008).

26. Hao, L. et al. Inductive sensor based on nano-scale SQUIDs. IEEE Trans. Appl. Supercond. 15, 514-517 (2005).

27. Gallop, J. SQUIDs: Some limits to measurement. Supercond. Sci. Technol. 16, 1575-1582 (2003)

28. Hao, L. et al. Novel methods of fabrication and metrology of superconducting nanostructures. IEEE Trans. Instrum. Meas. 56, 392-395 (2007).

29. Usadel, K. D. Generalized diffusion equation for superconducting alloys. Phys. Rev. Lett. 25, 507-509 (1970).

30. Wolf, E. L. Principles of Electron Tunneling Spectroscopy (Oxford Univ. Press, 1985).

\section{Acknowledgements}

We gratefully acknowledge O. Astafiev, L. Faoro, R. Fazio, M. E. Gershenson, T. T. Heikkilä, L. B. Ioffe, V. Piazza, P. Pingue, F. Portier, H. Pothier, H. Rabani, F. Taddei and A. S. Vasenko for fruitful discussions. The work was partially supported by the INFM-CNR Seed project 'Quantum-Dot Refrigeration: Accessing the $\mu \mathrm{K}$ Regime in Solid-State Nanosystems', and by the NanoSciERA project 'NanoFridge'.

\section{Author contributions}

F.G. conceived and carried out the experiment, analysed the data, carried out the calculations and wrote the manuscript. M.M. took part in the early stage of measurements, contributed to the cryogenic set-up and to writing the manuscript. J.T.P. designed and fabricated the samples, and contributed to writing the manuscript. J.P.P. took part in the early stage of measurements, contributed to the cryogenic set-up, took part in the interpretation of the data and contributed to writing the manuscript. F.G. and J.P.P. discussed the results and implications and commented on the manuscript at all stages equally.

\section{Additional information}

The authors declare no competing financial interests. Reprints and permissions information is available online at http://npg.nature.com/reprintsandpermissions. Correspondence and requests for materials should be addressed to F.G. 


\section{ADDENDUM}

\section{Superconducting quantum interference proximity transistor}

Francesco Giazotto, Joonas T. Peltonen, Matthias Meschke \& Jukka P. Pekola

Nature Physics 6, 254-259 (2010); published online: 1 April 2010; corrected after print: 10 June 2010.

This paper presents the realization of a superconducting quantum interference device that uses the superconducting proximity effect to achieve higher sensitivity in the measurement of magnetic fields than similar devices based on conventional Josephson junctions. It has also been drawn to our attention that we had inadvertently neglected to cite some prior works that described ${ }^{1,2}$ a related device known as an Andreev interferometer. We apologize for this oversight.

1. Petrashov, V. T., Antonov, V. N., Delsing, P. \& Claeson, T. Phase controlled mesoscopic ring interferometer. JETP Lett. 59, 551-555 (1994).

2. Petrashov, V. T., Antonov, V. N., Delsing, P. \& Claeson, T. Phase controlled conductance of mesoscopic structures with superconducting "mirrors". Phys. Rev. Lett. 74, 5268-5271 (1995). 